\title{
Colonic Health in Hospitalized Horses Treated with Non-Steroidal Anti-Inflammatory Drugs - A Preliminary Study
}

\author{
G van Galen ${ }^{\mathrm{a}, 1, *}$, C. Saegerman ${ }^{\mathrm{b}}$, S. Hyldahl Laursen ${ }^{\mathrm{a}, 2}$, S. Jacobsen ${ }^{\mathrm{a}}$, M. Andersson Munk ${ }^{\mathrm{a}}$, \\ H. Sjöströma , S. Holm Lindmark ${ }^{a}$, D. Verwilghen ${ }^{a, 1}$ \\ ${ }^{a}$ Large Animal Teaching Hospital, Department of Large Animal Science, University of Copenhagen, Denmark \\ ${ }^{\mathrm{b}}$ Research Unit of Epidemiology and Risk Analysis applied to veterinary sciences (UREAR-ULg), Fundamental and Applied Research for Animal and Health \\ (FARAH) Center, Faculty of Veterinary Medicine, University of Liege, Belgium
}

\section{A R T I C L E I N F O}

\section{Article history:}

Received 9 December 2020

Received in revised form 15 March 2021

Accepted 15 March 2021

Available online 26 March 2021

\section{Keywords:}

NSAID

Right dorsal colitis

Right dorsal colon

Fecal occult blood

Ultrasonography

\begin{abstract}
A B S T R A C T
Non-steroidal anti-inflammatory drugs (NSAIDs) can cause right dorsal colitis, but longitudinal clinical studies are lacking. This study investigates whether NSAID treated horses develop right dorsal colonic pathology in a clinical setting. Non-gastrointestinal hospitalized horses treated with NSAIDs $>4$ days, and untreated hospital-owned teaching horses and non-gastrointestinal client-owned hospitalized horses were included. All horses were monitored over time with clinical examinations (focusing on presence of colic, depression, reduced appetite, unstructured feces), ultrasonographic intestinal wall measurements, fecal occult blood tests (semi-quantitative results), and blood analysis (total protein and albumin concentrations, white blood cell and neutrophil counts). Outcomes were recorded as "ultrasonographically thickened right dorsal colon (RDC) walls", "colitis" and "right dorsal colitis". Findings over time were compared to baseline values and to control horses. Seventeen NSAID treated horses and 5 controls were included. NSAID treated horses developed thickened RDC walls (4/9), and subclinical and mild colitis (9/11) and right dorsal colitis (4/10), whereas all control horses remained healthy. The first changes were identified on treatment day 2. RDC walls of treated horses were significantly thicker compared to their own baseline values and compared to control horses. In conclusion, presumptive colon pathology was identified with a high incidence, starting early in the course of treatment, but with low severity. Appropriate monitoring should be advised throughout NSAID treatment. Additional research for noninvasive diagnostic tests for colon pathology is required.
\end{abstract}

(C) 2021 Elsevier Inc. All rights reserved.
Abbreviations: FOBT, fecal, occult blood test; ICS, intercostal space; NSAID, nonsteroidal anti-inflammatory drug; RDC, right dorsal colon; TRDC, thickening of the RDC wall; TP, serum total protein; WBC, white blood cell count.

Conflict of Interest: The study was partially funded by Equidan Denmark and Boehringer Ingelheim Denmark. The authors declare that they have no further conflict of interest.

Animal Welfare / Ethical Statement: Owner informed consent was obtained for client-owned horses and the hospital ethical commission approved the use of the teaching horses prior to study entry. The study was approved by the local ethical committee. Animals only underwent noninvasive diagnostics that improved monitoring. Blood samples on client-owned were only sampled when the horse required this for clinical reasons or when horses had a catheter.

* Corresponding author at: Gaby van Galen, DVM, PhD, Dipl ECEIM, Dipl ECVECC, The University of Sydney Australia

E-mail address: gaby@equinespecialists.eu (G. van Galen).

1 present address: Camden Equine Centre, University Teaching Hospital Camden, University of Sydney, Australia

2 present address: Malaren Equine Hospital, Sigtuna, Sweden

\section{Introduction}

Following treatment with non-steroidal anti-inflammatory drugs (NSAID's) side effects such as renal toxicity, gastric ulceration and right dorsal colitis can develop [1]. These insults to the right dorsal colon (RDC) are typically characterized as protein-losing enteropathy with RDC wall thickening and diarrhea [1]. Severe and advanced cases are described [2-4], but there is paucity in case descriptions of mild and subclinical right dorsal colitis. Nevertheless, there is good reason to believe that these mild and subclinical cases exist and are not uncommon following NSAID treatment.

Experimental studies where healthy horses are administered phenylbutazone have demonstrated the development of right dorsal colitis, with hypoproteinemia and neutropenia developing as early as 3-5 days after initiation of treatment with $8.8 \mathrm{mg} / \mathrm{kg}$ BWT q24H PO [5], and with $2.2 \mathrm{mg} / \mathrm{kg}$ BWT q12H PO [6]. Moreover, in vitro changes in colon tissue from healthy horses as result of NSAID's have been described, confirming the association between 
treatment and complications [7-9]. Although providing valuable information in controlled settings, experimental and in vitro studies do not reflect a clinical setting. Additional harmful effects on the colon can be exerted on patients by for example hospitalization stress, change of feeding, changes in appetite, side effects of other therapies, and nosocomial disease. These can increase incidence and severity and shorten the time of onset of which clinical signs of right dorsal colitis can appear. On the other hand, nowadays different and less toxic NSAID's than phenylbutazone are commonly used, possibly limiting toxicity.

Currently longitudinal observational studies on NSAID treated horses in a hospitalized environment are lacking. Therefore, the objective of this preliminary study is to obtain a preliminary idea of whether horses treated with NSAID's in a clinical setting develop signs that can be interpreted as presence of right dorsal colonic toxicity. Subobjectives are to determine its incidence, severity and onset time, and to compare RDC wall thickness during treatment to baseline values and to non-treated controls.

\section{Materials and Methods}

Case Selection

NSAID Horses

Client-owned horses admitted to the equine teaching hospital of the University of Copenhagen between March $25^{\text {th }}$ to June $2^{\text {nd }}$, and August $17^{\text {th }}$ to December $11^{\text {th }} 2015$ and matching the following criteria were included:

- > 4 months old,

- planned to stay hospitalized and receive NSAID treatment for at least 4 consecutive days, regardless of NSAID type, administration route or dose,

- no previous NSAID treatment during the last month (but horses receiving NSAIDs within $24 \mathrm{H}$ before study enrollment were accepted),

- hospitalized for reasons other than intestinal disease or investigation,

- no colic or colitis in the last month prior to study entry,

- fecal egg count $<500$ epg.

Horses were monitored daily, starting the morning after the first NSAID dose, until the day after the last NSAID administration, provided they had not been discharged at this time. If possible, cases with thickening of the right dorsal colon (TRDC) were followed daily until thickening subsided.

\section{Control Horses}

Client-owned horses admitted between August $17^{\text {th }}$ to December $11^{\text {th }} 2015$ to the equine teaching hospital of the University of Copenhagen and with the following criteria were included:

- > 4 months old,

- planned to be hospitalized without receiving NSAID treatment for at least 7 consecutive days,

- not treated with NSAIDs during the last month,

- hospitalized for reasons other than intestinal disease or investigation,

- no colic or colitis in the last month prior to study entry,

- fecal egg count < 500 epg.

Horses were monitored daily, starting the morning following admission, and until discharge or until initiation of NSAID treatment.

In addition, 4 adult hospital-owned teaching horses were included in the control group. They had not received NSAID's or suffered from colic or colitis during the last month prior to study entry. Two of them were newly acquired teaching horses, mimicking the environmental change and stress experienced by hospitalized horses. The two others were randomly chosen from the teaching herd. The teaching horses were followed for 14 days while being in full teaching activity.

\section{Data Collection}

Signalment, the primary condition (diagnosis, evolution), NSAID treatment (drug, administration route, dosage, duration) and other therapies were recorded. Every day horses were examined with focus on appetite, depression and colic. Fecal consistency was scored daily (1: dry and hard, 2: hard, 3: normal moist, 4: very moist, but with structure, 5: soft without structure, cow pie, 6: runny, watery). A fecal occult blood test (FOBT) ${ }^{\mathrm{a}}$ was performed daily on fresh, warm feces collected from the ground. Results were read after 5 minutes, always in a similar room and time, and using a semi-quantitative scale $(0$ : negative, no color of test line, 1 : very pale and difficult to see test line, 2: pale but clearly visible test line, 3: test line has similar color as control line, 4: test line darker than control line; personal communication N. Kerbyson and own unpublished data). Positive albumin and/or hemoglobin results obtained within $24 \mathrm{H}$ after rectal examination and positive hemoglobin results after upper respiratory tract surgery or bleeding were considered false positives and were discarded. Ultrasonographic measurements of the RDC wall were obtained in intercostal space (ICS) 10,11, 12, 13 and 14 with a portable ultrasound scanner $^{\mathrm{b}}$ and a $4.5 \mathrm{MHz}$ curvilinear transducer ${ }^{\mathrm{c}}$. Horses were not sedated or clipped, and alcohol was applied to improve contact. At every ICS two measurements were taken, each on different still images (referred to as "coupled measurements"). The mean value calculated from those coupled measurements was used for the study. Besides the RDC, also the duodenum, jejunum, left colon, right ventral colon and cecum were scanned. Ultrasonography was performed daily on NSAID treated horses and client-owned control horses; every other day on teaching horses. In client-owned horses with an intravenous catheter and in teaching horses, blood was standardly sampled and analyzed on admission and then repeated daily. Serum total protein (TP) and albumin, white blood cell count (WBC) and neutrophil count were measured in an external laboratory.

Owner informed consent was obtained for client-owned horses and the hospital ethical commission approved the use of the teaching horses prior to study entry. The study was approved by the local ethical committee of the University of Copenhagen.

\section{Definitions of Outcomes}

TRDC was defined as an ultrasonographic measure of the RDC wall that has at least three out of the four following criteria:

1) a minimal percentual increase from baseline (this increase will be determined by the largest percentual increase of control horses),

2) a minimal absolute increase from baseline (this increase will be determined by the mean maximal increase from baseline values of control horses, and by the largest difference between coupled measurements from control cases),

3) > the highest baseline value measured,

4 ) $\geq$ mean +3 SD of baseline values calculated per localization.

A horse had TRDC if these criteria were identified on at least one of the locations (ICS $10,11,12,13$ or 14 ) on at least one day.

Colitis (regardless of the section of the large intestine, but possibly involving the RDC) was defined as presence of at least two of the four following criteria:

1) TRDC as defined above, or thickening of another part of the large intestine visualized through ultrasonography. 
Table 1

Signalment of NSAID treated horses and control horses.

\begin{tabular}{llllll}
\hline & & \multicolumn{2}{l}{ NSAID treated horses $(n=17)$} & \multicolumn{2}{l}{ Control horses $(n=5)$} \\
& & $\mathrm{N}$ & $\%$ & $\mathrm{~N}$ & $\%$ \\
\hline \multirow{2}{*}{ Sex } & Mare & 8 & $47 \%$ & 4 & $80 \%$ \\
& Gelding & 8 & $47 \%$ & 1 & $20 \%$ \\
\multirow{3}{*}{ Breed } & Stallion & 1 & $6 \%$ & 0 & $0 \%$ \\
& Saddle horse & 11 & $65 \%$ & 5 & $100 \%$ \\
& Pony & 2 & $12 \%$ & 0 & $0 \%$ \\
& Icelandic horse & 4 & $23 \%$ & 0 & $0 \%$ \\
Age (years) & & Mean \pm SD (range) & Mean \pm SD (range) \\
& & $8.8 \pm 5.3(2-21)$ & $9.8 \pm 3.8(6-14)$ \\
\hline
\end{tabular}

Abbreviation: NSAID = non-steroidal anti-inflammatory drug; $\mathrm{N}=$ number of horses; $\mathrm{SD}=$ standard deviation.

Table 2

Overview of ultrasonographic measurements of the right dorsal colon wall of NSAID treated horses and control horses. Mean, SD and range are calculated out of the measures obtained for every horse per intercostal space (ICS10, ICS11, ICS12, ICS13 and ICS14).

\begin{tabular}{|c|c|c|c|c|c|c|c|}
\hline & \multicolumn{3}{|c|}{ NSAID treated horses $(n=9)$} & \multicolumn{3}{|c|}{ Control horses $(n=5)$} & \multirow{2}{*}{$\begin{array}{l}\text { Two-sample t test with } \\
\text { unequal variances ( } P \text { value })\end{array}$} \\
\hline & mean & SD & range & mean & SD & range & \\
\hline $\begin{array}{l}\text { Difference between coupled } \\
\text { measurements }(\mathrm{cm})\end{array}$ & 0.03 & 0.02 & $0.0-0.12$ & 0.02 & 0.02 & $0.0-0.05$ & 0.36 \\
\hline Baseline values $(\mathrm{cm})$ & 0.20 & 0.03 & $0.14-0.29$ & 0.21 & 0.02 & $0.16-0.24$ & 0.41 \\
\hline Minimal values $(\mathrm{cm})$ & 0.18 & 0.02 & $0.13-0.24$ & 0.17 & 0.01 & $0.14-0.19$ & 0.15 \\
\hline Maximal values $(\mathrm{cm})$ & 0.29 & 0.09 & $0.19-0.51$ & 0.22 & 0.02 & $0.20-0.27$ & $0.004^{*}$ \\
\hline $\begin{array}{l}\text { Maximal absolute increase from } \\
\text { baseline (maximal value - baseline } \\
\text { value; } \mathrm{cm} \text { ) }\end{array}$ & 0.09 & 0.08 & $0.0-0.33$ & 0.01 & 0.01 & $0.0-0.04$ & $0.0004^{*}$ \\
\hline $\begin{array}{l}\text { Maximal percentual increase from } \\
\text { baseline (maximal value / baseline; \%) }\end{array}$ & $146 \%$ & $42 \%$ & $100-283 \%$ & $105 \%$ & $7 \%$ & $100-125 \%$ & $0.0006^{*}$ \\
\hline $\begin{array}{l}\text { Maximal absolute increase from } \\
\text { minimal values (maximal value - } \\
\text { minimal value; } \mathrm{cm} \text { ) }\end{array}$ & 0.11 & 0.08 & $0.03-0.33$ & 0.05 & 0.02 & $0.02-0.09$ & $0.005^{*}$ \\
\hline $\begin{array}{l}\text { Maximal percentual increase from } \\
\text { minimal value (maximal value / } \\
\text { minimal value; \%) }\end{array}$ & $158 \%$ & $42 \%$ & $116-283 \%$ & $128 \%$ & $11 \%$ & $111-157 \%$ & $0.008^{*}$ \\
\hline
\end{tabular}

Abbreviation: NSAID = non-steroidal anti-inflammatory drug; SD = standard deviation; ${ }^{*}=$ significantly different $(P<0.05)$.

2) A high grade FOBT (grade 3 or 4 ) for at least two consecutive days. Positive albumin and/or hemoglobin results obtained $24 \mathrm{H}$ after a rectal examination and positive hemoglobin results after upper respiratory tract surgery or bleeding were considered false positives and were discarded.

3) Decrease in one or more of the following blood values, unexplained by treatments or primary disease: TP (decrease at least $10 \%$ of baseline value and $\leq 60 \mathrm{~g} / \mathrm{L}$ ), albumin (decrease at least $10 \%$ of baseline value and $\leq 30 \mathrm{~g} / \mathrm{L}$ ), WBC count (decrease at least $20 \%$ of baseline value and $\leq 5^{*} 10^{9} / \mathrm{L}$ ), neutrophil count (decrease at least $20 \%$ of baseline value and $\leq 2.5 * 10^{9} / \mathrm{L}$ ),

4) Development of at least one of the following clinical signs, not directly explicable by the primary disease: colic, depression, reduced appetite and unstructured feces (grade 5 or 6 ) $[1,4,10]$.

Right dorsal colitis was defined with the same criteria as colitis, but with certitude of RDC involvement through detection of TRDC and absence of thickening of other intestinal sections visualized with ultrasonography.

According to the appearance of the clinical signs, the horses with evidence of colitis or right dorsal colitis were grouped as subclinical or clinical.

\section{Data Analysis}

\section{Descriptive Statistics}

Mean, SD and range are used to describe continuous variables from all horses. The number of horses with a response and with a positive response and percentages are used to describe categorical variables.

\section{Statistical Analysis}

Variables of NSAID horses were compared to those of control horses. Comparisons of qualitative parameters were performed using a logistic regression. Comparisons of quantitative parameters were performed using a two-sample Wilcoxon ranksum (MannWhitney) test and by assuming an unequal variance and nonnormal data distribution [11]. For the ultrasonographic wall thickness of the RDC over time, each time point was compared with its baseline and with the corresponding time point of the control group using a Welch's test for unequal variances. Frequencies and proportions were compared with Fisher's exact test [12]. $P$ values $<0.05$ were considered significant. Commercial statistical software was used. ${ }^{\mathrm{d}}$

\section{Results}

\section{Case Description}

Seventeen NSAID treated horses, one client-owned control horse and 4 hospital-owned control horses were included in the study. Details on signalment are provided in Table 1 . Reasons for hospitalization of NSAID treated horses were: tongue lesion (1), traumatic wound on limbs (8), upper respiratory tract or sinus surgery (5), esophageal obstruction (1), corneal ulceration (1) and arthroscopy (1). The client-owned control horse was hospitalized for 12 days for extensive poor performance and lameness work up and ophthalmological investigation.

Horses were fed 2-3 times per day according to individually adjusted feeding plans with hay and concentrated feeds. Horses were fasted prior to surgery. 
A

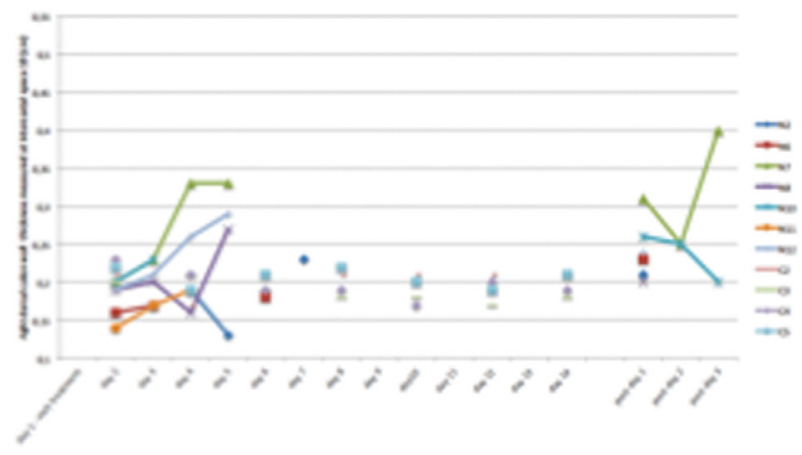

C

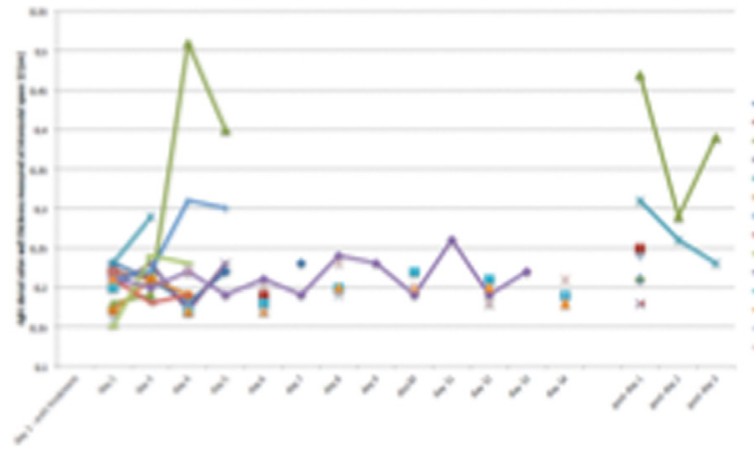

$\mathrm{E}$

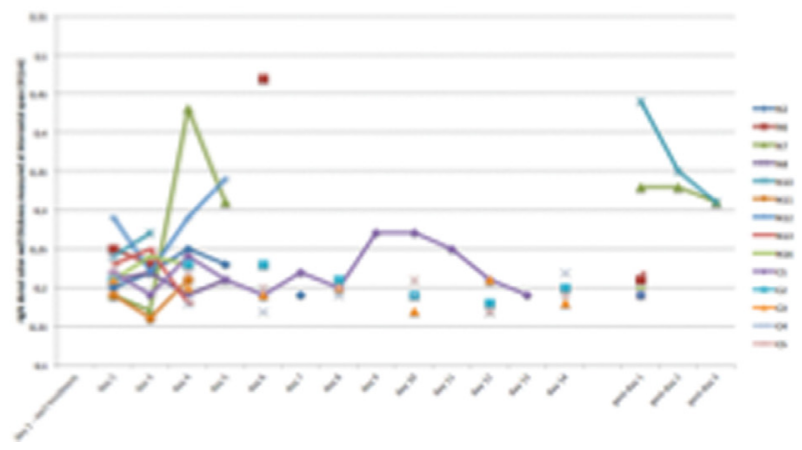

B

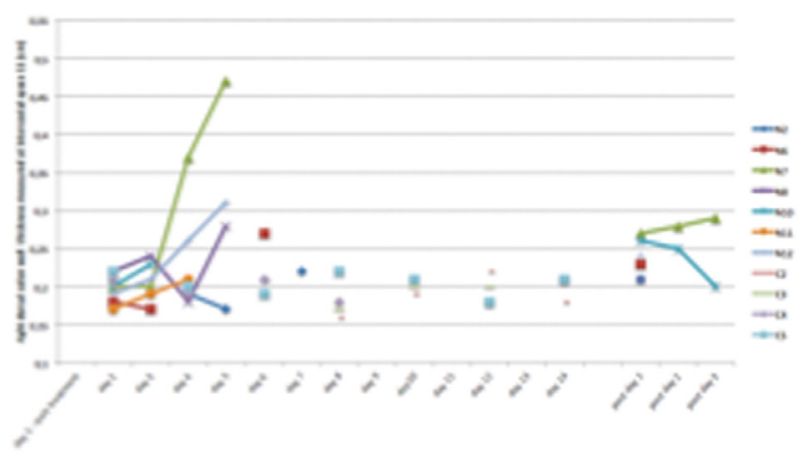

D

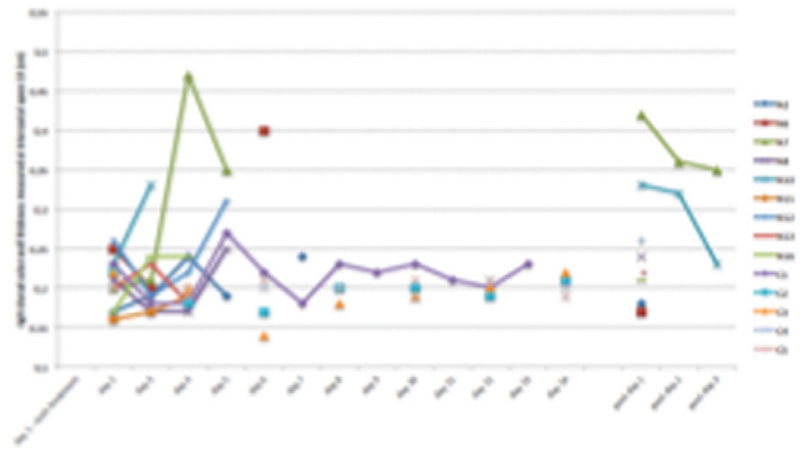

Fig. 1. a-e Ultrasonographic wall thickness of the right dorsal colon of 9 NSAID treated horses and 5 untreated control horses measured at intercostal space $10-14$.

\section{NSAID Treatment}

Horses received intravenous flunixin ${ }^{\mathrm{e}}$ (8), intravenous followed by oral flunixin ${ }^{f}(4)$, intravenous flunixin ${ }^{\mathrm{e}}$ followed by oral meloxicam ${ }^{g}(4)$ or oral meloxicam ${ }^{g}$ followed by intravenous flunixin $^{\mathrm{e}}$ (1). NSAID treatment was administered for $6.1 \pm 2.4$ days (range 4-13 days). Thirteen horses received at least one of the treatment days more flunixin than labeled $(1.3-2.2 \mathrm{mg} / \mathrm{kg}$ instead of $1.1 \mathrm{mg} / \mathrm{kg}$ ). Meloxicam administration was never administered above the labeled dose $(0.6 \mathrm{mg} / \mathrm{kg})$. Additionally, two horses received topical diclofenacdiethylamin ${ }^{\mathrm{h}}$ for 1 and 2 days.

\section{Other Treatments}

Fifteen of the 17 NSAID horses received systemic antimicrobials: penicillin $^{\mathrm{i}, \mathrm{j}}$ (15), gentamycin ${ }^{\mathrm{k}}$ (7) and sulfadiazine/trimethoprim ${ }^{\mathrm{l}}$ (1). Twelve underwent surgery: 11 under general anesthesia and 1 standing. Thirteen horses received at least once a sedation unrelated to the surgical intervention. The client-owned control horse received local ophthalmological treatment and an intra-articular injection. One hospital-owned control horse was sedated once.

\section{Clinical Signs and Fecal Consistency}

Control horses did not develop clinical signs, maintained good appetite, normal fecal consistency and normal demeanor. Several NSAID treated horses developed mild clinical signs that were not explicable by the primary disease: mild colic (4), depression (4), increased fecal consistency grade ( 3 with grade 5 ), reduced appetite (3), or combinations of these clinical signs (4).

\section{Fecal Occult Blood Test}

None of the horses showed consistently a negative FOBT throughout their follow up period. Six high-grade albumin results of control horses and 2 high-grade albumin and 2 high-grade hemoglobin results of NSAID treated horses were discarded because of rectal examinations taking place in the 24 hours prior to the test. In 7 NSAID treated horses, hemoglobin results were considered false positives because of upper respiratory tract or sinus bleeding.

For the remaining FOBT results, control horses only showed an occasional, single-day grade 3 for hemoglobin (2) and/or albumin (4), and never grade 4 . One control horse had a grade 3 albumin result for 3 consecutive days. In NSAID treated horses, grades 3 and 

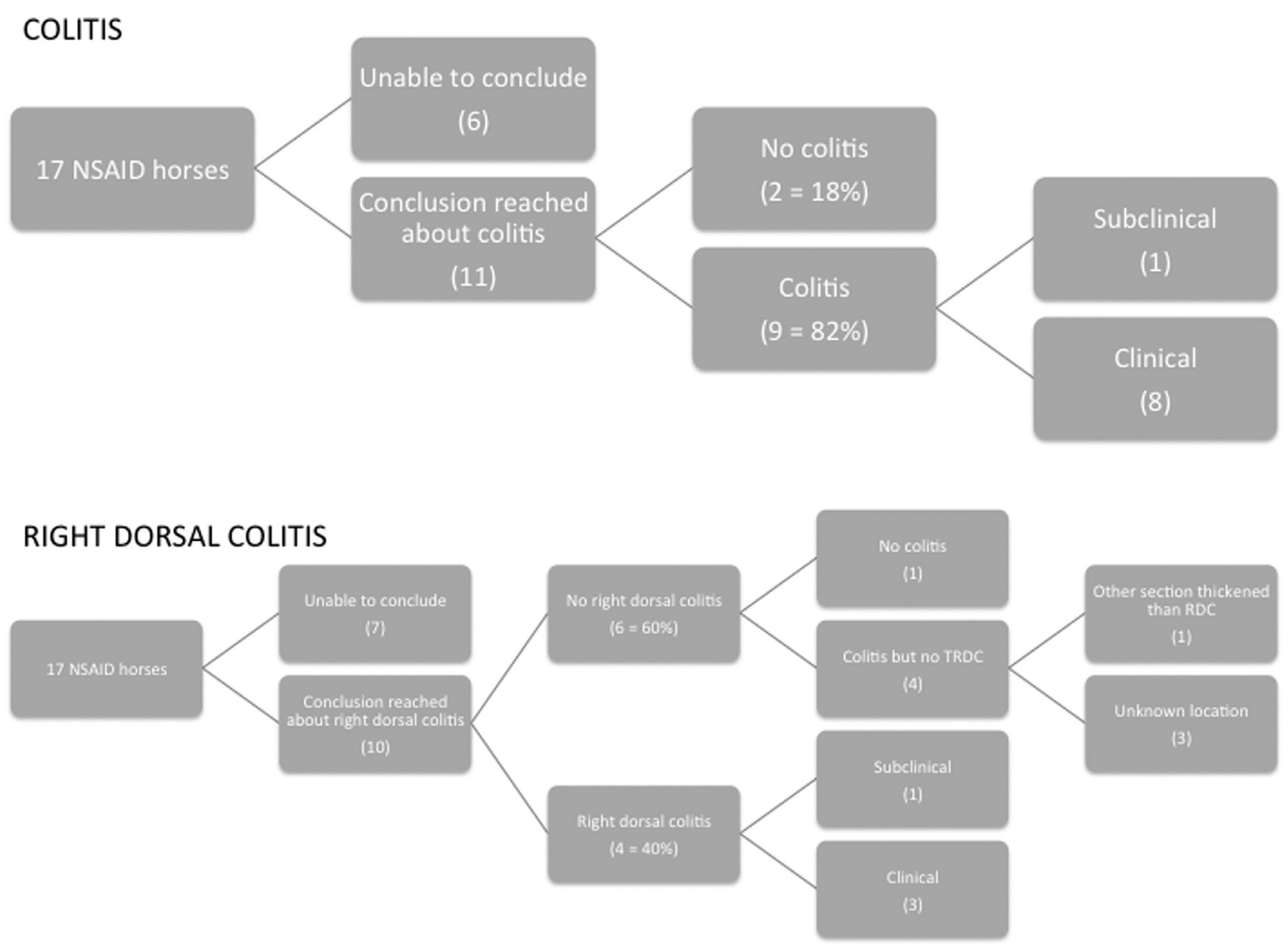

Fig. 2. Grouping of NSAID horses with regards to colitis and right dorsal colitis definitions.

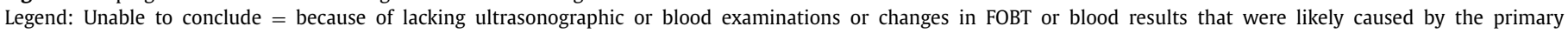
condition or treatment.

4 were commonly encountered for hemoglobin (single day in 5/10 cases; several days in a row in 2/10 cases) and/or albumin (single day in 15/17 cases; several days in a row in 9/17 cases).

\section{Ultrasonography and TRDC}

Eight of the 17 NSAID treated cases could not be scanned due to thick hair coat or dangerous behavior. Based on the ultrasonographic measurements (Table 2), the definition of TRDC has been set as an ultrasonographic measure of the RDC wall that has at least three out of the four following criteria:

1) minimal percentual increase: $157 \%$ of baseline value,

2) minimal absolute increase: $0.05 \mathrm{~cm}$,

3) $>0.29 \mathrm{~cm}$,

$4) \geq$ mean +3 SD of baseline values calculated per localization.

Four out of the 9 scanned NSAID treated horses (44\%) developed TRDC whereas control horses remained all with a normal RDC wall thickness. TRDC was detected as early as day 3 of treatment in 1 horse, on day 4 in 2 horses and day 6 in one horse. During post treatment monitoring, RDC wall measurements decreased again (Fig. 1 - 3).

In one horse (case 16) no TRDC developed, but a mild left colonic (baseline $0.21 \mathrm{~cm}$, peak $0.35 \mathrm{~cm}$ ) and jejunal thickening (baseline $0.17 \mathrm{~cm}$, peak $0.33 \mathrm{~cm}$ ) was noted. No other horses developed thickening of other gastro-intestinal sections than the RDC.

\section{Blood Analysis}

Due to absence of a catheter, one control horse and 6 treated horses could not be sampled throughout their treatment period. The 4 remaining control horses showed no changes in blood parameters. In one NSAID treated horse all 4 parameters dropped at day 13 (TP $48.3 \mathrm{~g} / \mathrm{L}$, albumin $27.9 \mathrm{~g} / \mathrm{L}$, WBC $2.2 * 10^{9} / \mathrm{L}$, neutrophils $\left.1.3^{*} 10^{9} / \mathrm{L}\right)$. Another NSAID treated horse developed neutropenia $\left(2.5^{*} 10^{9} / \mathrm{L}\right)$ on day 4 . These findings were unrelated to the primary conditions (traumatic wound).

\section{Colitis and Right Dorsal Colitis}

None of the control horses showed indications of colitis or right dorsal colitis. Nine horses $(9 / 11=82 \%$, including all 4 horses with TRDC) fulfilled the criteria for the colitis definition and 4 $(4 / 10=44 \%$, including all 4 horses with TRDC) for the right dorsal colitis definition (Fig. 2 and 3). Time of onset of recorded changes was variable amongst horses, but the first changes (typically depression and FOBT positive results) were identified as early as day 2 of NSAID treatment.

\section{Statistical Comparison}

Sex and age were not different between treated and nontreated groups. Maximal RDC wall thickness, maximal increases from baseline, and maximal increases from minimal values during treatment from treated horses were significantly higher than those of control horses (Table 2). At treatment day 2, RDC walls from treated horses were significantly thinner compared to control horses in ICS space 10 and 11 ( $P 0.009$ and $P$ 0.04, respec- 


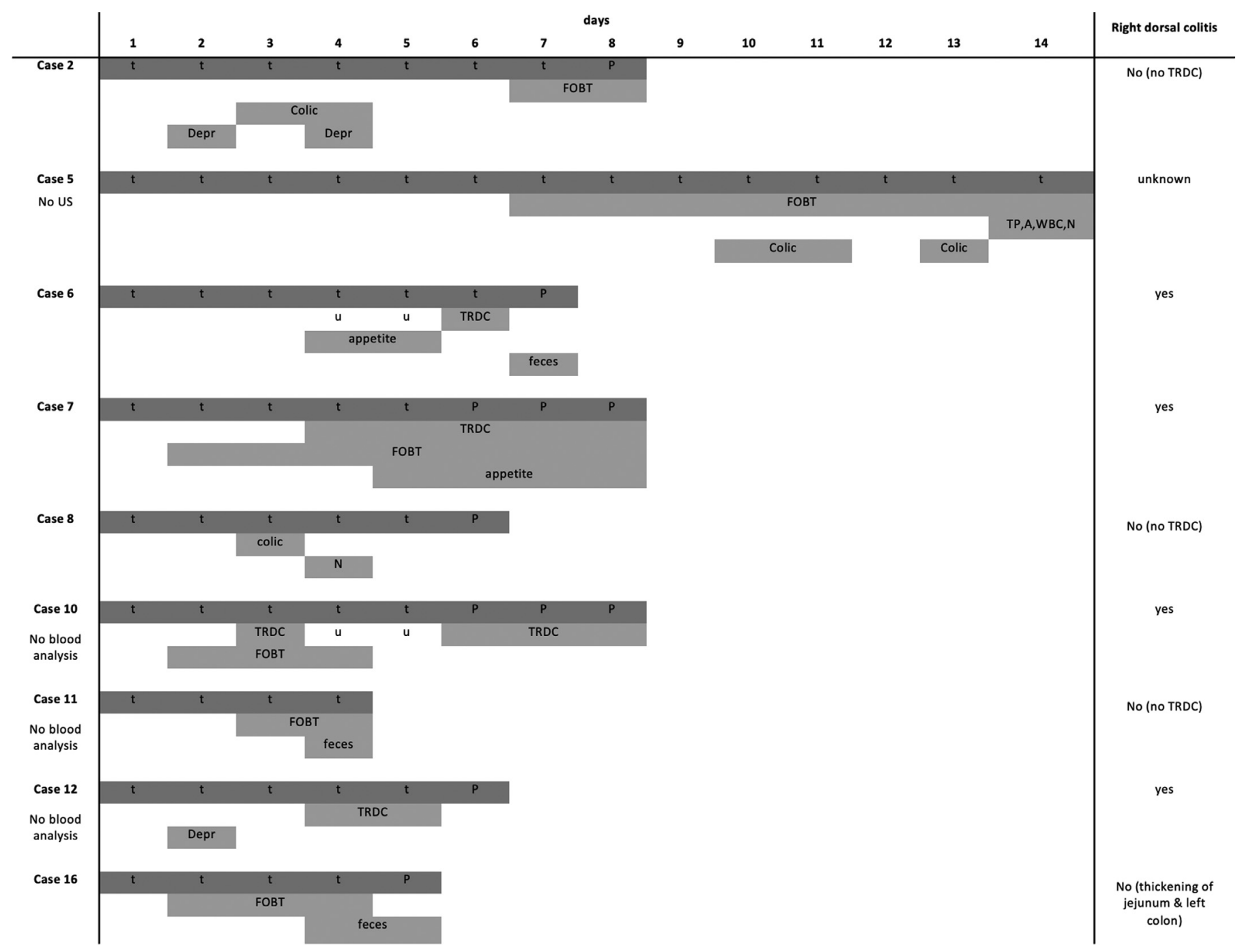

Fig. 3. Criteria and timing of development of colitis following NSAID treatment.

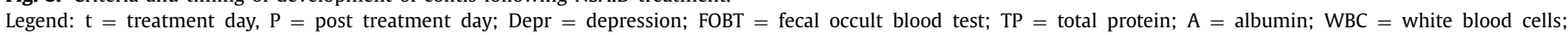
$\mathrm{N}=$ neutropenia; appetite = lack of appetite; feces = unstructured feces (grade 5 or 6 ).

tively). However, they were significantly thicker compared to con-

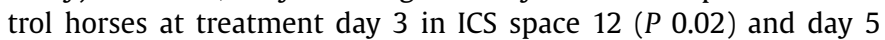
in ICS space 10,11 and $14(P 0.008, P 0.047$ and $P 0.05$, respectively). At day 5 they were also significantly thicker compared to their own baseline values in ICS space 10 and $11(P<0.001$ and $P$ 0.05 , respectively; Fig. 4). The noted changes in clinical signs, blood parameters and FOBT results showed no statistically differences between groups.

\section{Discussion}

In contrast to earlier experimental studies, this is the first longitudinal study describing the effects of NSAID's on the equine colon in a clinical setting. It shows a high incidence, yet low severity, of right dorsal colitis developing early in the course of treatment. Clinical studies are inherently complicated by absence of a gold standard for diagnosing right dorsal colitis and absence of a clear definition of right dorsal colitis in literature. As a result, for the present study three outcomes (TRDC, colitis and right dorsal colitis) were included to discern different forms of colon pathology. Interestingly, TRDC always occurred in conjunction with clinical signs, and can therefore be suggested a strong indicator for right dorsal colitis. This is also in line with the current existing litera- ture, where the diagnosis of right dorsal colitis is typically made ultrasonographically by detecting TRDC [1-2]. Not all cases defined as having colitis had TRDC and thus could not be concluded to suffer from right dorsal colitis, some of which were due to missing ultrasonographic data. In these cases important differential diagnoses were antimicrobial induced colitis, nutritional changes, stress and hospital acquired infections. All can give rise to clinical signs, changes in blood analysis and changes in FOBT similar to right dorsal colitis. Also thickening of colon walls can occur, however, thickening of only the RDC wall, as is typically seen in right dorsal colitis, would be unlikely.

Unfortunately, in several NSAID treated cases no outcome (TRDC, colitis or right dorsal colitis) could be concluded because of absent or temporarily unreliable complementary examinations, underestimating the incidences of colon pathology in this study. Still, TRDC and subclinical or mild clinical colitis and right dorsal colitis were identified in a several of NSAID treated horses. This was done through use of indicators of colon pathology that are widely and easily available in practice: clinical signs, FOBT, abdominal ultrasonography and blood analysis. The clinical signs used and detected in this study (colic, depression, reduced appetite and unstructured feces) are nonspecific, especially when mild, can be associated to many different conditions, and cannot be used to dif- 

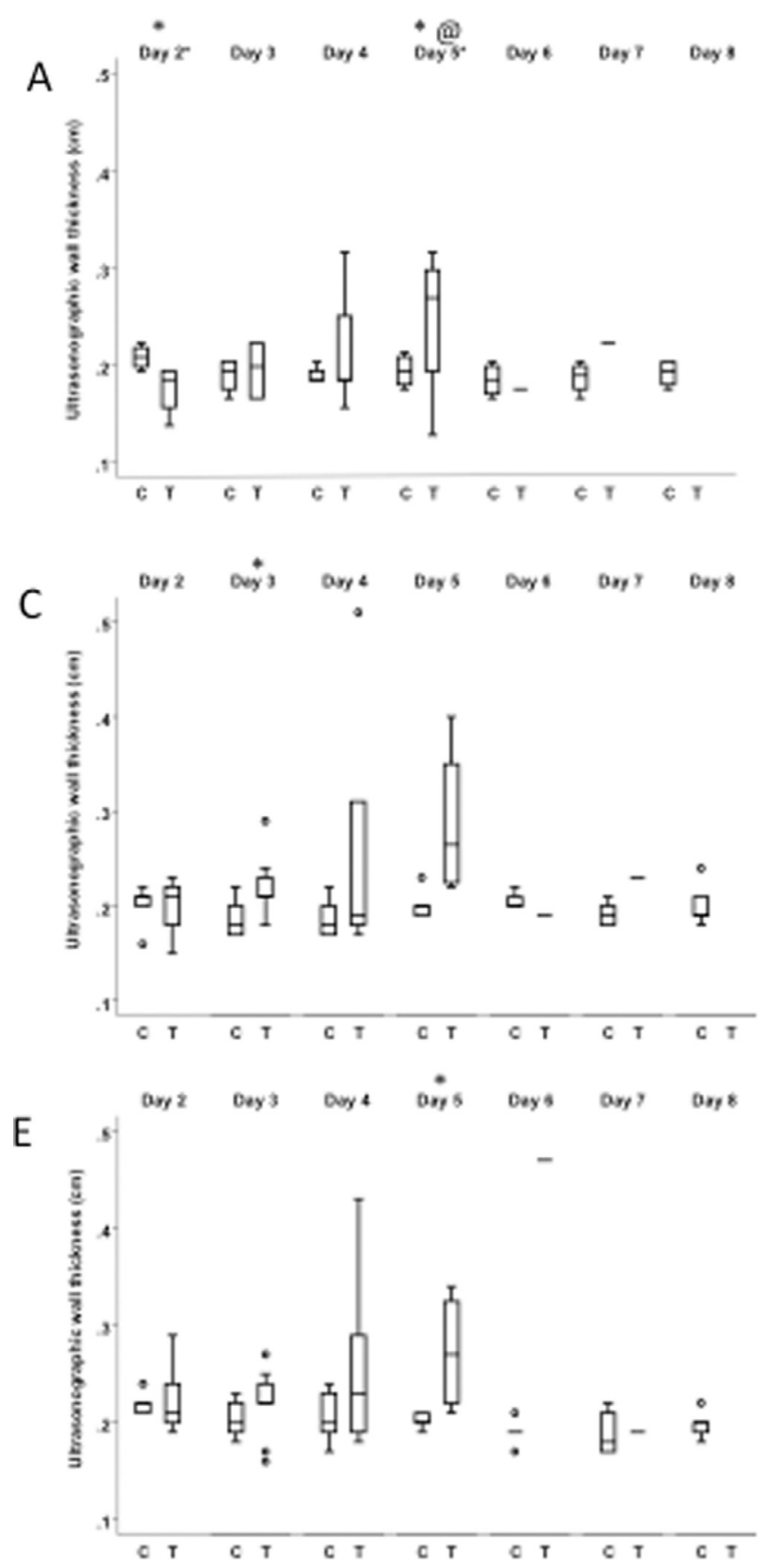

B
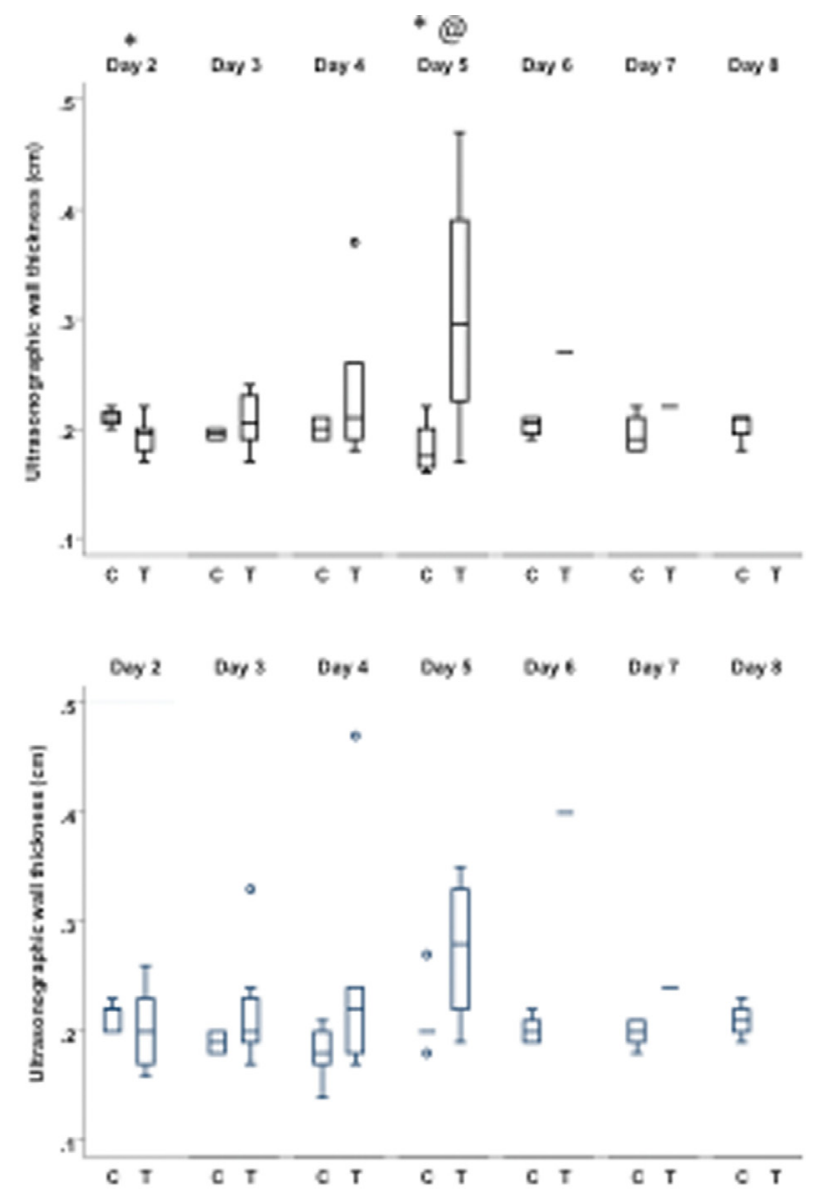

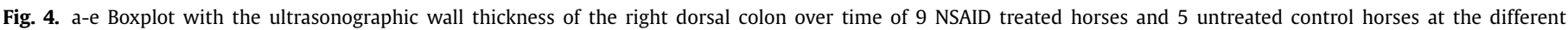
intercostal spaces (ICS): ICS10 (A), ICS11 (B), ICS12 (C), ICS13 (D), ICS14 (E).

Legend: $\mathrm{C}=$ Control horses; $\mathrm{T}=$ Treated horses; ${ }^{*}=$ significant difference between $\mathrm{C} \& \mathrm{~T}$;

@ = significant difference between T baseline \& T day x. Significance is set at $P<0.05$.

ferentiate between different forms of colitis. Moreover, they might have introduced bias in this study, because they can be triggered by the primary disease or by other side effects of NSAID therapy such as gastric ulcers. To reduce bias as much as possible, colitis and right dorsal colitis definitions were never based on clinical signs alone and clinical signs were only used when they were not explicable by the primary disease. Despite the above-mentioned difficulties, clinical signs are of major importance to determine severity of colon affection (subclinical - mild - severe).

The FOBT allowed differentiating between groups by serial testing and the use of the semi-quantitative test result read- ing, especially for albumin. It should be recognized that positive hemoglobin results can originate from lesions from the upper respiratory tract and/or the entire length of the gastro-intestinal tract, including from gastric ulcers. Positive albumin results, on the other hand, are believed to be caused by lesions in the large intestines.

In the present study the repeatability of ultrasonographic measurements was not assessed with statistical methods, but was esteemed good based on the small differences between the coupled measurements. These differences were also found to be similar to those previously described for other intestinal sections [13]. Since intestinal wall measurements vary significantly between studies 
[13], the current study defined TRDC on self-established criteria and compared to baseline values. Some horses with TRDC had a wall thickness only slightly above or even within reported reference values. Similar cases may easily be overlooked in practice without comparison to baseline values and/or in the absence of serial measurements throughout NSAID treatment. The ultrasonographic criteria developed and used in this study to detect TRDC (minimal percentual increase of $157 \%$ of baseline value over time, minimal absolute increase of $0.05 \mathrm{~cm}$ over time, and a thickness of $>0.29 \mathrm{~cm}$ at any moment), can be suggested as new ultrasonographic guidelines for clinical use. Furthermore, false negatives of TRDC or right dorsal colitis can occur because of invisibility of the contralateral RDC wall, or ante-rupture thinning [2]. Ultrasonography is believed helpful to diagnose overt right dorsal colitis [2], and appeared valuable in the current study to detect subclinical and mild cases.

In previous experimental studies, decreases in plasma albumin concentration $[5,6,14]$, plasma protein concentration $[6,14]$ and neutrophil count [5] were detected following NSAID treatment. Also, many clinicians only confirm a diagnosis of right dorsal colitis when there is presence TRDC together with a decline in albumin concentrations. In this study important declines were only found in 2 treated horses, suggesting that right dorsal colitis, especially subclinical, can occur without significant drop in albumin concentrations. Interfering factors prohibiting detection of declines are inflammatory changes related to the primary disease, fluid administration, primary disease or complications, rendering the use of blood parameters less helpful in clinical settings.

Overall, this study emphasizes that the use and interpretation of indicators of RDC pathology is not always straightforward. However, our results support the use of baseline values and serial measurements for all applied indicators. The first changes in the current study could be detected as early as day 2 of treatment and TRDC as from day 3. This time of onset is similar or slightly earlier than recorded in previous experimental studies where findings were detected earliest at day 3 [5] or day 5 [6]. In contrast to previous studies [2-6], no severe disease occurred in any of the horses. This might be explained by the use of less toxic NSAID's (flunixin and meloxicam). Despite the mild clinical impact, the incidence of gastrointestinal side effects was high though.

Limitations of this study include absence of a diagnostic gold standard for right dorsal colitis, some missing or temporarily unreliable complementary examinations and the fact that the study was not executed in a blind manner. Another limitation is also the small and heterogenous study population with a relatively short duration of NSAID treatment, and the small control group. However, this study was only meant as a preliminary study to develop a better understanding about whether colon problems are occurring in a clinical population treated with NSAIDs. Despite its many limitations, this study delivers interesting information about changes in colon health over time in hospitalized horses treated with NSAIDs. Moreover, one should be cautious to conclude that the changes in colon health in the treated horses are caused by NSAIDs as this study is an observational study and is not evaluating causation, and differences in severity of disease between control and treatment groups or treatment differences, could have affected outcome. The findings of this study need to be confirmed and further studied in depth with future larger clinical studies that will address the shortcomings of the current study.

\section{Conclusions}

In conclusion, a high incidence of mild colon pathology was encountered. Changes examinations that are assessing colon health were detected after as early as 2 days of NSAID treatment. Studies including a larger study population and longer treatment du- ration are needed to confirm these findings. NSAID's are widely used and necessary drugs in equine medicine, but considering the side effects we recommend appropriate monitoring. Especially ultrasonography revealed useful, and new guidelines of ultrasonographically assessing right dorsal colon thickness can be suggested based on this study. Future research should be aimed to develop additional non-invasive diagnostic modalities and to confirm these findings.

\section{Acknowledgements}

Nicola Kerbyson and Derek Knottenbelt are gratefully acknowledged for sharing their experiences with the Succeed $₫$ equine fecal occult blood test. Also, all equine clinicians in working on the study cases are warmly thanked.

\section{Funding}

The study was funded by the SCIL / EVECCS grant, Equidan and Boehringer Ingelheim Denmark.

\section{Manufacturer's Details}

a Succeed ${ }^{\circledR}$ fecal occult blood test, Freedom Health LLC., Aurora, US

b Portable ultrasound scanner, GE Healthcare Vivid I, Horten, Norway

c Transducer, GE Healthcare Vivid I, Horten, Norway

d Stata/SE 14, Statacorp LP, College Station, Texas

e Finadyne ${ }^{\circledR}$ Vet., solution for injection, 50mg/ml, MSD Animal Health, Intervet International B.V., Boxmeer, The Netherlands

${ }^{f}$ Finadyne ${ }^{\circledR}$ Vet., oral pasta, MSD Animal Health, Intervet International B.V., Boxmeer, The Netherlands

g Metacam ${ }^{\circledR}$, oral suspension $15 \mathrm{mg} / \mathrm{ml}$, Boehringer Ingelheim Vetmedica GmbH, Ingelheim/Rhein, Germany

h Diclofenac ${ }^{\circledR}$, topical gel $11.6 \mathrm{mg} / \mathrm{g}$, Orifarm Generics $A / S$, Odense S, Denmark

i Benzylpenicillin, solution for injection, Panpharma, Luitré, France

j Penovet ${ }^{\circledR}$ Vet., solution for injection, $300000 \mathrm{IU} / \mathrm{ml}$, Boehringer Ingelheim, Copenhagen, Denmark

k Genta-Equine ${ }^{\circledR}$, solution for injection, $100 \mathrm{mg} / \mathrm{ml}$, Franklin Pharmaceutical Limited, Trim, Co Meath, Ireland

${ }^{1}$ Equibactin ${ }^{\circledR}$ Vet., oral paste, 45 gram, Dechra, Oudewater, The Netherlands

\section{Author Statement}

van Galen G.: Conceptualization, methodology, formal analysis, investigation, resources, data curation, writing - original draft, writing - review and editing, visualization, supervision, project administration, funding acquisition

Saegerman C.: formal analysis, writing - review and editing, visualization

Hyldahl Laursen S.: investigation, writing - review and editing, visualization

Jacobsen S.: Conceptualization, writing - review and editing, supervision

Andersson Munk M.: formal analysis, investigation, writing original draft

Sjöström $H$. : formal analysis, investigation, writing - original draft

Holm Lindmark S.: formal analysis, investigation, writing - original draft

Verwilghen D.: Conceptualization, methodology, writing - review and editing 


\section{References}

[1] Cook VL, Blikslager AT. The use of nonsteroidal anti-inflammatory drugs in critically ill horses. J vet emerg crit care 2015;25:76-88.

[2] Jones SL, Davis J, Rowlingson K. Ultrasonographic findings in horses with right dorsal colitis: five cases (2000-2001). J Am Vet Med Assoc 2003;222:1248-51.

[3] East LM, Trumble TN, Steyn PF, Savage CJ, Dickinson CE, Traub-Dargatz JL. The application of technetium-99m hexamethylpropyleneamine oxime (99mTc-HMPAO) labeled white blood cells for the diagnosis of right dorsal ulcerative colitis in two horses. Vet radiol ultrasound 2000;41:360-4.

[4] Hough ME, Steel CM, Bolton JR, Yovich JV. Ulceration and stricture of the right dorsal colon after phenylbutazone administration in four horses. Aust vet $\mathrm{j}$ $1999 ; 77: 785-8$

[5] McConnico RS, Morgan TW, Williams CC, Hubert JD, Moore RM. Pathophysiologic effects of phenylbutazone on the right dorsal colon in horses. American j vet res 2008;69:1496-505.

[6] Reed SK, Messer NT, Tessman RK, Keegan KG. Effects of phenylbutazone alone or in combination with flunixin meglumine on blood protein concentrations in horses. Am j vet res 2006;67:398-402.

[7] Meschter CL, Gilbert M, Krook L, Maylin G, Corradino R. The effects of phenylbutazone on the intestinal mucosa of the horse: a morphological, ultrastructural and biochemical study. Equine vet j 1990;22:255-63.
[8] Campbell NB, Jones SL, Blikslager AT. The effects of cyclo-oxygenase inhibitors on bile-injured and normal equine colon. Equine vet j 2002;34:493-8.

[9] Richter RA, Freeman DE, Wallig M, Whittem T, Baker GJ. In vitro anion transport alterations and apoptosis induced by phenylbutazone in the right dorsal colon of ponies. Am j vet res 2002;63:934-41.

[10] Collins LG, Tyler DE. Phenylbutazone toxicosis in the horse: a clinical study. ] Am Vet Med Assoc 1984;184:699-703.

[11] Dagnelie P. Statistique théorique et appliquée. Inférence statistique à une et à deux 474 dimensions. Belgique: Bruxelles; 1998.

[12] Petrie A, Watson PF. Statistics for Veterinary and Animal Science. 3rd edn; 2013. p. 393.

[13] Bithell S, Habershon-Butcher JL, Bowen IM, Hallowell GD. Repeatability and reproducibility of transabdominal ultrasonographic intestinal wall thickness measurements in thoroughbred horses. Vet radiol ultrasound 2010;51:647-51.

[14] MacAllister CG, Morgan SJ, Borne AT, Pollet RA. Comparison of adverse effects of phenylbutazone, flunixin meglumine, and ketoprofen in horses. J Am Vet Med Assoc 1993;202:71-7. 